\title{
OPEN Characterization of hyperglycemia due to sub-chronic administration of red ginseng extract via comparative global proteomic analysis
}

\author{
Ann-Yae $\mathrm{Na}^{1}$, Jung Jae Jo ${ }^{1}$, Oh Kwang Kwon ${ }^{1}$, Piljoung Cho ${ }^{1}$, Yan Gao ${ }^{1}$, Ju-Hyun Kim²,
} Kyu Min Kim ${ }^{3}$, Sung Hwan $\mathrm{Ki}^{3 \otimes}$ \& Sangkyu Lee ${ }^{1 \bowtie}$

Ginseng (Panax ginseng Meyer) is commonly used as an herbal remedy worldwide. Few studies have explored the possible physiological changes in the liver although patients often self-medicate with ginseng preparations, which may lead to exceeding the recommended dose for long-term administration. Here, we analyzed changes in the hepatic proteins of mouse livers using quantitative proteomics after sub-chronic administration of Korean red ginseng (KRG) extract (control group and $0.5,1.0$, and $2.0 \mathrm{~g} / \mathrm{kg} \mathrm{KRG)} \mathrm{using} \mathrm{tandem} \mathrm{mass} \mathrm{tag} \mathrm{(TMT)} \mathrm{6-plex} \mathrm{technology.} \mathrm{The} 1.0$ and $2.0 \mathrm{~g} / \mathrm{kg} \mathrm{KRG}$ groups exhibited signs of liver injury, including increased levels of aspartate transaminase (AST) and alanine aminotransferase (ALT) in the serum. Furthermore, serum glucose levels were significantly higher following KRG administration compared with the control group. Based on the upregulated proteins found in the proteomic analysis, we found that increased cystathionine beta-synthase (CBS) and cystathionine gamma-lyase (CSE) levels promoted greater hydrogen sulfide $\left(\mathrm{H}_{2} \mathrm{~S}\right)$ synthesis in the liver. This investigation provides novel evidence that sub-chronic administration of KRG can elevate $\mathrm{H}_{2} \mathrm{~S}$ production by increasing protein expression of $\mathrm{CBS}$ and CSE in the liver.

Ginseng (Panax ginseng Mayer), is commonly been used as an herbal remedy in traditional medicine-it can be air-dried to produce white ginseng, or steamed or heated to produced red ginseng in order to preserve it and enhance its efficacy ${ }^{1,2}$. Korean red ginseng (KRG) and its components, such as ginsenosides, have been associated with various beneficial pharmacological effects on blood pressure, atherosclerosis, and hyperlipidemia by reducing oxidative damage ${ }^{3-5}$. Although $\mathrm{KRG}$ is considered safe for ingestion and tests on the safety of ginseng preparations have been conducted using animals, the US Food and Drug Administration has reported adverse effects arising from overexposure to functional foods including ginseng ${ }^{6,7}$. Recently, it has been reported that ginseng can damage vascular smooth muscle ${ }^{8}$ and lead to contractile dysfunction in vascular smooth muscle ${ }^{9}$; however, despite its long history and broad use, few studies have explored physiological changes in the liver after sub-chronic high-dose administration of ginseng.

The liver is a representative organ that detoxifies various absorbed xenobiotics, synthesizes proteins, and produces several biochemicals in mammalians for indispensable physiological functions. The liver, especially, is one of the main organs that supplies circulating blood and, consequently various tissues, with glucose ${ }^{10}$. Changes in blood glucose concentration may also occur depending on liver dysfunction. Furthermore, physiological changes in the liver are commonly caused by the use of exogenous compounds such as drugs, herbs, and alcohol ${ }^{11}$. These liver toxicities are well screened clinically with indicators such as aspartate transaminase (AST) and alanine aminotransferase (ALT) $)^{12}$, and the two indicators are used to directly judge the effect of ginseng on liver function ${ }^{13}$.

In this investigation, we show that significantly higher cystathionine beta-synthase (CBS) and cystathionine gamma-lyase (CSE) levels promote greater hydrogen sulfide $\left(\mathrm{H}_{2} \mathrm{~S}\right)$ synthesis in the liver after sub-chronic administration of high-dose KRG. $\mathrm{H}_{2} \mathrm{~S}$ is thought to be an endogenously produced gaseous signaling molecule, similar

${ }^{1}$ BK21 FOUR Community-Based Intelligent Novel Drug Discovery Education Unit, College of Pharmacy and Research Institute of Pharmaceutical Sciences, Kyungpook National University, Daegu 41566, Republic of Korea. ${ }^{2}$ College of Pharmacy, Yeungnam University, Gyeongsan 38541, Republic of Korea. ${ }^{3}$ College of Pharmacy, Chosun University, Gwangju 61452, Republic of Korea. ${ }^{\varpi}$ email: shki@chosun.ac.kr; sangkyu@knu.ac.kr 
A

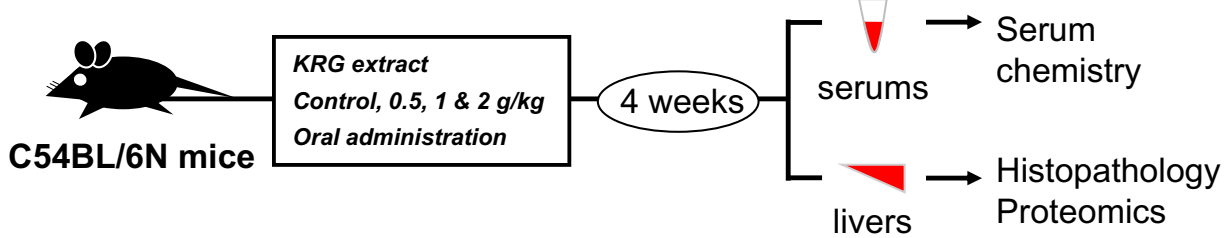

B

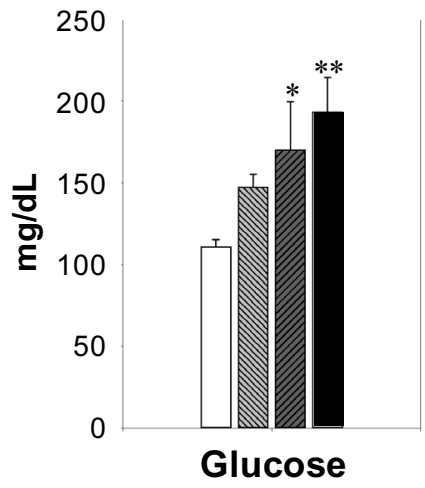

C

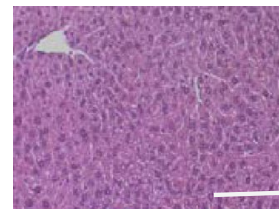

Control

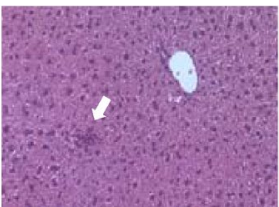

$\mathrm{KRG} 0.5 \mathrm{~g} / \mathrm{kg}$
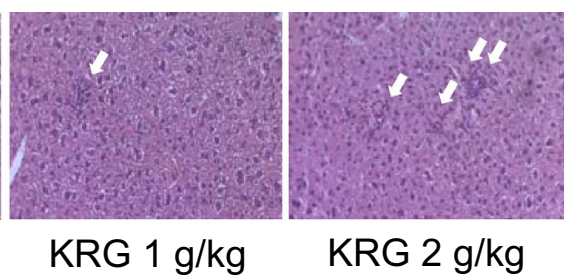

Figure 1. Overview of the experimental approach. Five-week-old C57BL/6 N male mice were orally administered different doses $(0.5 \mathrm{~g}, 1.0 \mathrm{~g}$, and $2.0 \mathrm{~g} / \mathrm{kg}$ ) of Korean red ginseng (KRG). After 4 weeks, serum and liver tissue were extracted for serum chemistry and proteomic analysis (A). Glucose levels and AST and ALT levels in the serum (B). Histological analysis of $\mathrm{H} \& \mathrm{E}$ staining of liver tissue at $200 \times$ magnification. White arrows indicate inflammatory cell infiltration. Scale bar represents $150 \mathrm{~mm}$. (C). Results are shown as the mean \pm SEM of five to six animals per group. Statistical significance was set at ${ }^{\star} p<0.05$ and ${ }^{\star *} p<0.01$ compared with the control based on an ANOVA (SPSS Statistics 23 software package, https://www.ibm.com/analytics/spssstatistics-software).

to nitric oxide (NO) and carbon monoxide, which plays a role in the regulation of inflammatory responses, apoptosis, oxidative stress, and angiogenesis ${ }^{14-17}$. Additionally, $\mathrm{H}_{2} \mathrm{~S}$ is reportedly related to the regulation of glucose metabolism ${ }^{18,19} \cdot \mathrm{H}_{2} \mathrm{~S}$ is now recognized as an important cellular signaling molecule due to its important functions in several aspects of human health and disease ${ }^{20}$. Despite the beneficial effects of KRG in various physiological states, the way KRG affects $\mathrm{H}_{2} \mathrm{~S}$ levels has not previously been explored. Considering the importance of the liver for $\mathrm{H}_{2} \mathrm{~S}$ production, it is important to explore how $\mathrm{H}_{2} \mathrm{~S}$ affects glucose metabolism and liver injury after long-term overexposed KRG administration. Thus, in this investigation, we explored the protein dynamics in the livers of mice after sub-chronic KRG administration using proteomic analysis.

\section{Results}

Changes in clinical characteristics. In general, the daily recommended oral doses of ginseng for rodents is $500 \mathrm{mg} / \mathrm{kg}$. These doses were calculated based on actual doses of red ginseng calculated for human beings $(1.5-3.0 \mathrm{~g} / \text { person/day })^{21,22}$. In this study, we administered KRG extract to mice orally every day for 28 days at a maximum of 4 times the recommended dose $(0.5,1.0$, and $2.0 \mathrm{~g} / \mathrm{kg})$ to investigate the clinical effects of highdose KRG (Fig. 1A). We quantified the ginsenoside contained in the KRG extract used in the experiment in our previous study ${ }^{23}$. These dosages have been used previously in general toxicity assessments in rodent models ${ }^{24,25}$. After 4 weeks of administration, we measured several serum parameters to characterize liver function. It was determined that glucose levels increased significantly, and serum AST and ALT levels were also significantly higher in the 1.0 and $2.0 \mathrm{~g} / \mathrm{kg} \mathrm{KRG}$ groups, respectively, which indicated liver injury (Fig. 1B). Furthermore, the hepatic pathologic change was confirmed in H\&E stained tissues (Fig. 1C). Histopathological results were dis- 
A
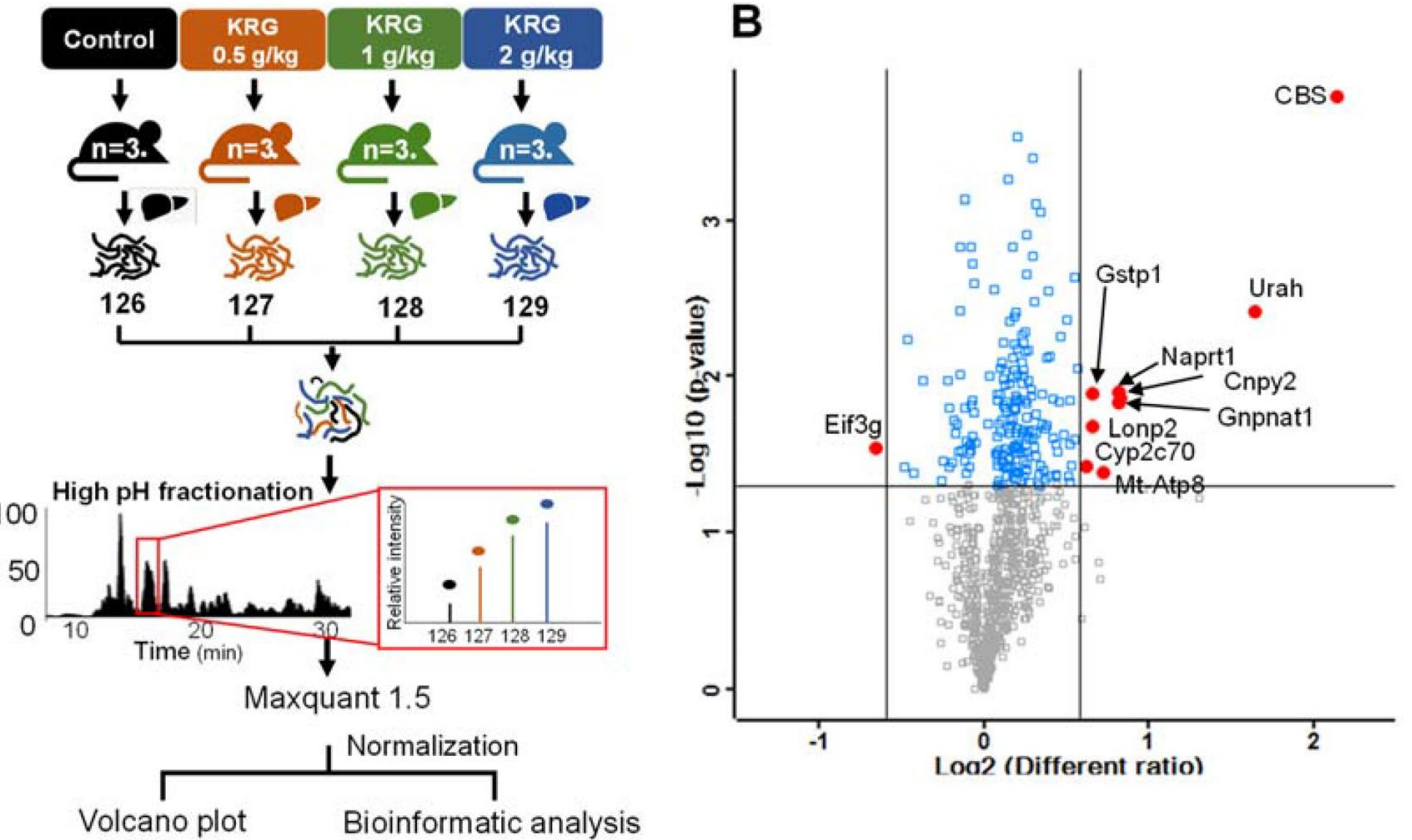

Figure 2. Overall scheme for the global proteomic profiling of mouse liver (A). Volcano plot and distribution of quantified proteins based on the reporter ion ratio in the three KRG groups (B).

tinctly shown focal inflammatory cell infiltration in $2 \mathrm{~g} / \mathrm{kg} \mathrm{KRG}$ group compare to control group (White arrow). The liver function test and lipid profile results are summarized in Table S1. There were no other significant differences in terms of body weight, liver weight, or other serum parameters between groups.

Global protein profiling in mice administered with KRG. The process used for protein profiling in this investigation is presented in Fig. 2A. In the 3 group, we identified a combined total of 1005 proteins, where 881 of them were quantified based on comparative proteomic analysis (Table S2). All data contained technical duplicates and the mass error for all identified peptides was assessed, with the highest mass error being $>0.02 \mathrm{Da}$ (Fig. S1A). We conducted a Pearson correlation analysis of the reporter ion intensities to determine the quantitative accuracy of MS-based proteomics, with a resulting of 0.8775 (Fig. S1B).

Classification of differentially expressed proteins after the administration of high-dose KRG. Proteins identified from the liver tissue of the $3 \mathrm{KRG}$ groups were assigned a reporter ion ratio over the control group for tandem mass tag (TMT) analysis to identify the differentially expressed proteins (DEPs) associated with KRG-induced liver injury. We identified a total of 58 DEPs after comparing the 3 KRG groups and the control group (Table S3). A total of 19, 40, and 31 DEPs were identified at the $0.5,1.0$, and $2.0 \mathrm{~g} / \mathrm{kg} \mathrm{KRG}$ groups, respectively ( $\log _{2}$ ratio $\geq 0.585$ (upregulated) or $\log _{2}$ ratio $\leq-0.585$ (down-regulated)), for each KRG group over the control group (Fig. S1C). In addition, we employed one-way $t$-tests with a $p$ value cutoff of 0.05 to identify proteins whose levels changed significantly after KRG administration. A Volcano Plot of DEP was illustrated by fold change ( $\log _{2}$ Difference) versus significance $(-\log 10 p$ value) using a threshold value of 0.05 . Protein IDs in red were considered significantly up- or down-regulated using the Perseus software package (Fig. 2B). Based on these criteria, 9 upregulated proteins showed statistical significance (Table 1).

Verification of cystathionine $\beta$-synthase (CBS) and cystathionine gamma-lyase (CSE) protein expression and $\mathrm{H}_{2} \mathrm{~S}$ levels. After exploring the role of each protein and its association with hyperglycemia after KRG administration for 4 weeks, we focused on the most upregulated protein, CBS, and validated the accuracy of the TMT-labeled quantitative proteomics results using Western Blot (Fig. S2A). Furthermore, we determined that CSE is also an important protein in this study even though the proteomics results include no upregulation of CSE in the KRG groups. This is explained by the abundance of CBS and CSE in the liver and their involvement in the endogenous production of $\mathrm{H}_{2} \mathrm{~S}$ and its metabolism ${ }^{26}$. CBS protein expression levels were found to be significantly higher in all KRG groups $(0.5 \mathrm{~g} / \mathrm{kg}$; KRG relative level to control $2.07(p<0.05), 1 \mathrm{~g} / \mathrm{kg}$ KRG; relative level to control $2.49(p<0.01), 2 \mathrm{~g} / \mathrm{kg} \mathrm{KRG}$; relative level to control $2.28(p<0.01))$, supporting the quantitative proteomics analysis. Also, the relative ratio of CSE protein expression was 1.6-fold $(p<0.01)$ which was increased in the only $2.0 \mathrm{~g} / \mathrm{kg} \mathrm{KRG}$ group (relative level to control, 1.0) (Fig. 3A). These changes in protein levels support the belief that dominant $\mathrm{H}_{2} \mathrm{~S}$-producing enzymes are highly expressed in the liver after high-dose KRG administration. When we measured the $\mathrm{H}_{2} \mathrm{~S}$ levels in the liver samples using ELISA assays to further characterize CBS and CSE proteins, a significant dose-dependent increase in $\mathrm{H}_{2} \mathrm{~S}$ concentration was seen in 1.0 and $2.0 \mathrm{~g} / \mathrm{kg} \mathrm{KRG}$ mouse liver tissues (3.1 umol/L, $(p<0.001) ; 3.5 \mathrm{umol} / \mathrm{L},(p<0.001))$ (Fig. 3B). 


\begin{tabular}{|c|c|c|c|c|c|c|c|c|c|}
\hline \multirow[b]{2}{*}{ No } & \multirow[b]{2}{*}{ Accession } & \multirow[b]{2}{*}{ Protein name } & \multirow[b]{2}{*}{ Gene name } & \multirow[b]{2}{*}{ peptides } & \multirow{2}{*}{$\begin{array}{l}\text { Protein } \\
\text { coverage } \\
(\%)\end{array}$} & \multicolumn{3}{|c|}{$\begin{array}{l}\text { Ratio (KRG/ } \\
\text { control) }\end{array}$} & \multirow[b]{2}{*}{$p$-value } \\
\hline & & & & & & 0.5 & 1.0 & 2.0 & \\
\hline 1 & Q91WT9 & Cystathionine beta-synthase & Cbs & 3 & 3.9 & 4.48 & 4.24 & 4.54 & 0.000 \\
\hline 2 & Q9CRB3 & 5-hydroxyisourate hydrolase & Urah & 2 & 13.6 & 2.73 & 3.21 & 3.48 & 0.004 \\
\hline 3 & Q8CC86 & Nicotinate phosphoribosyltransferase & Naprt1 & 6 & 12.8 & 1.67 & 1.64 & 2.02 & 0.013 \\
\hline 4 & Q9JK38 & $\begin{array}{l}\text { Glucosamine 6-phosphate } N \text {-acetyltrans- } \\
\text { ferase }\end{array}$ & Gnpnat1 & 2 & 7.1 & 1.58 & 1.74 & 2.01 & 0.015 \\
\hline 5 & Q9QXT0 & Protein canopy homolog 2 & Cnpy2 & 2 & 14.3 & 1.55 & 1.88 & 1.92 & 0.014 \\
\hline 6 & Q7JCZ0 & ATP synthase protein 8 & mt-Atp8 & 2 & 34.3 & 1.39 & 2.00 & 1.65 & 0.040 \\
\hline 7 & P19157 & Glutathione $S$-transferase P 1 & Gstp1 & 8 & 41 & 1.42 & 1.70 & 1.63 & 0.014 \\
\hline 8 & Q9DBN5 & Lon protease homolog 2, peroxisomal & Lonp2 & 2 & 2.6 & 1.54 & 1.80 & 1.42 & 0.022 \\
\hline 9 & Q91W64 & Cytochrome P450 2C70 & Сур2с70 & 5 & 10.8 & 1.73 & 1.61 & 1.30 & 0.038 \\
\hline
\end{tabular}

Table 1. List of statistically significant upregulated proteins.

A

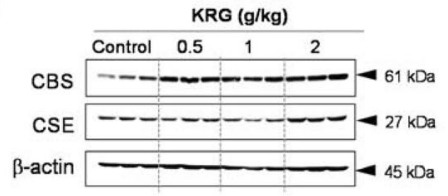

C

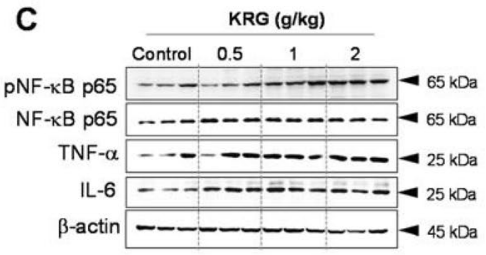

D

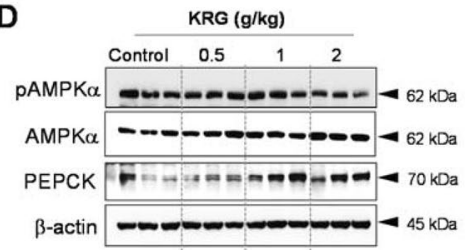

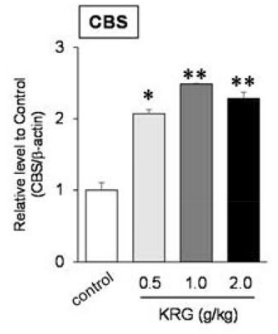

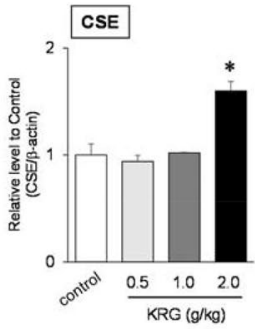

B
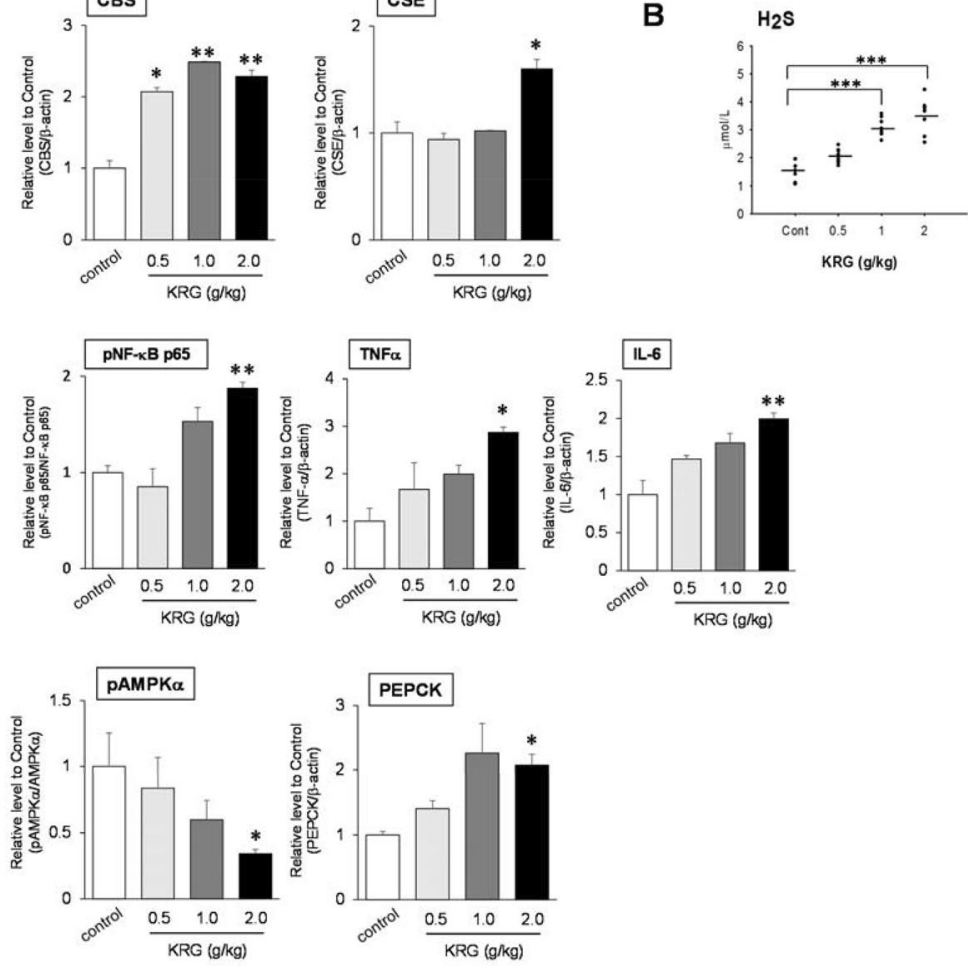

Figure 3. Verification of the changes in protein level in mouse liver following the administration of highdose concentrations of KRG. Cystathionine $\beta$-synthase (CBS) and cystathionine $\gamma$-lyase (CSE) protein levels compared to $\beta$-actin via Western blots (A). $\mathrm{H}_{2}$ S production based on ELISA (B). Changes in protein levels with increased $\mathrm{H}_{2} \mathrm{~S}$ and the effects on liver injury $(\mathbf{C})$ and the gluconeogenesis pathway (D) in mouse liver following the administration of high-dose concentrations of KRG. Bands were quantified using a densitometer in the ImageJ program, and the $\mathrm{H}_{2} \mathrm{~S}$ results are expressed as mean $\pm \mathrm{SE}(n=5-6)$. Statistical significance was set at * $p<0.05,{ }^{* *} p<0.01$, and ${ }^{* * *} p<0.001$ compared with the control based on an ANOVA (SPSS Statistics 23 software package, https://www.ibm.com/analytics/spss-statistics-software).

Protein expression levels and the association with oxidative stress and hyperglycemia through $\mathrm{H}_{2}$ S. Phospho-NF- $\mathrm{kB}$ p65, NF- $\mathrm{kB}$, TNF- $\alpha$, and IL-6 expression levels were measured using Western Blot analysis to assess the inflammatory response to KRG over-dose (Fig. S2B). A significant increase in NF- $\mathrm{BB}$ p65 (relative level to control 1.88, $(p<0.01)$ ), TNF- $\alpha$ (relative level to control 2.87, $(p<0.05)$ ), and IL-6 (relative level to control 1.99, $(p<0.01)$ ) was seen in the $2.0 \mathrm{~g} / \mathrm{kg} \mathrm{KRG}$ group compared to the control group (relative level to control 1.0) (Fig. 3C). Furthermore, to explore the role of $\mathrm{H}_{2} \mathrm{~S}$ in the liver in terms of gluconeogenesis, we assessed the protein levels of phospho-5' AMP-activated protein kinase alpha (pAMPKa), AMPKa, and PEPCK, which are involved in the gluconeogenesis metabolic pathway (Fig. S2C). High levels of $\mathrm{H}_{2} \mathrm{~S}$ activity in the liver 


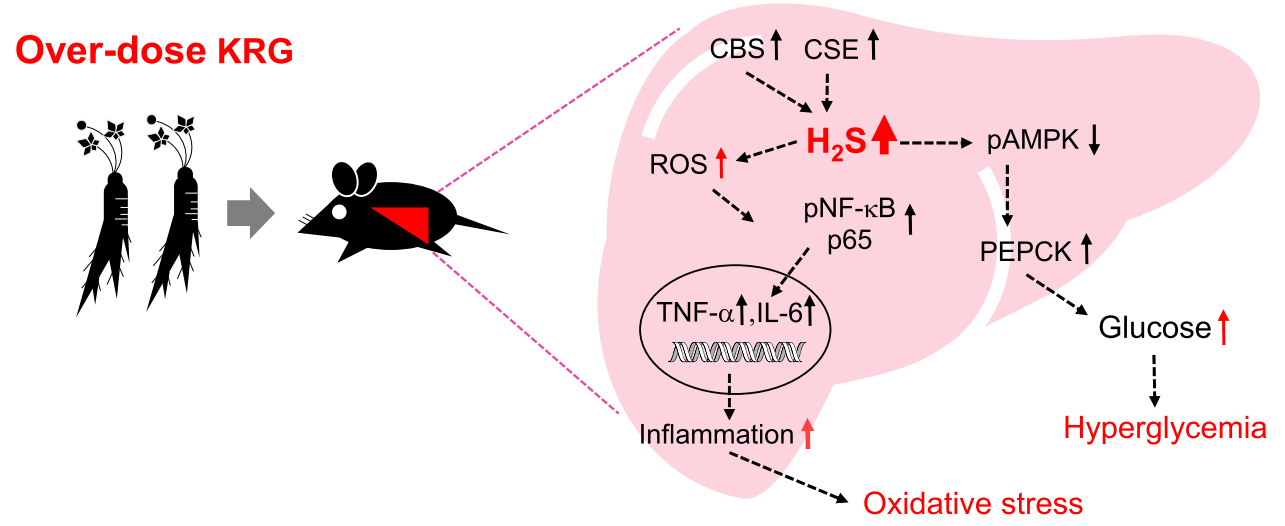

Figure 4. Signaling pathways underlying KRG-induced oxidative stress and hyperglycemia through $\mathrm{H}_{2} \mathrm{~S}$.

dose-dependently inhibited the expression of phosphorylated AMPKa over AMPKa, with the $2.0 \mathrm{~g} / \mathrm{kg}$ KRG group showing an almost $40 \%$ decrease in phospho-AMPKa/AMPKa protein levels (relative level to control 0.34, $(p<0.05)$ ) (Fig. 3D). Interestingly, high-dose KRG increased PEPCK protein levels (2 g/kg KRG; relative level to control 2.81, $(p<0.05)$ ) by increasing $\mathrm{H}_{2} \mathrm{~S}$ levels. In summary, we demonstrated that KRG over-dose contributes to $\mathrm{H}_{2} \mathrm{~S}$ synthesis, resulting in increased oxidative stress in the liver and hyperglycemia (Fig. 4).

Gene ontology and Kyoto encyclopedia of genes and genomes pathway analysis of the DEPs based on bioinformatics. We analyzed the gene ontology (GO) and Kyoto Encyclopedia of Genes and Genomes (KEGG) pathways by calculating the Fisher's exact test $p$ value for 47 upregulated DEPs in order to increase our understanding of the mechanisms by which the sub-chronic administration of KRG in 3 groups, at different doses, led to liver injury. Fig. S3 summarizes the top five annotations from the Fisher exact tests for each category. The GO-based distribution of upregulated proteins was also evaluated to characterize the liver injury caused by sub-chronic high-dose KRG administration in mouse liver tissue. The KEGG pathway analysis showed that steroid hormone biosynthesis and sulfur metabolism were significantly enhanced with KRG administration. The domain annotation enrichment from the InterPro results indicated that cytochrome P450 was present at significantly higher concentrations in mouse liver after receiving high doses of KRG.

\section{Discussion}

It has long been believed that ginseng is a nontoxic herbal medicine, but investigations have been conducted to determine its safety. Most of the previous ginseng research focused only on its protective effects as a medicinal herb for the treatment of a variety of medical condition $s^{27,28}$. In this investigation, we showed for the first time that high levels of $\mathrm{H}_{2} \mathrm{~S}$ may play an important role in regulating oxidative stress and hyperglycemia levels in the liver by sub-chronic high-dose KRG administration. To explore the mechanisms underlying the negative effects of high-dose KRG, an in vivo model was used for proteomic analysis with TMT labeling technology. We demonstrated that sub-chronic administration of high doses of KRG caused high serum AST and ALT levels and elevated blood glucose (Fig. 1B). Although we couldn't find reference studies be comparable to subchronic administration of high dose KRG to normal mice, previous studies on liver function decline following long-term administration or high-dose administration for 90 days were confirmed. For examples, subchronic administration for 90 days of the main KRG metabolite of ginseng saponin, compound $\mathrm{K}$, in beagle dogs showed increased ALT level in groups with compound $\mathrm{K}^{29}$. And a single case study reported KRG $6 \mathrm{~g} /$ day dose for 12 weeks, the liver function test showed increased AST and ALT level compared to prior liver enzymes ( 6 month earlier $)^{30}$. These previous results could be suggest possibilities of negative effects on liver enzymes due to sub chronic high-dose KRG administration.

Although rare, as can be seen from the case of acute hepatotoxicity after consumption of ginseng-related products, the need for mechanistic research on hepatotoxicity derived from ginseng used as a health functional food is proposed. We listed 47 upregulated proteins to determine the biological mechanisms of KRG-induced hepatotoxicity. Interestingly, CBS was the most upregulated protein in all the KRG groups (Table 1) and $\mathrm{H}_{2} \mathrm{~S}$-generating reactions were catalyzed by CBS and CSE in the trans-sulfuration pathway ${ }^{31}$. Thus, the hyperglycemia caused by sub-chronic administration in the high-dose KRG groups may be associated with increased $\mathrm{H}_{2} \mathrm{~S}$ synthesis via $\mathrm{CBS}$ and CSE in the liver, given that CSE is an important $\mathrm{H}_{2} \mathrm{~S}$-producing enzyme that can be upregulated by $\mathrm{NO}^{26}$. Several earlier studies that explored $\mathrm{KRG}$ treatment and its association with $\mathrm{H}_{2} \mathrm{~S}$ showed, for example, that $\mathrm{KRG}$ treatment inhibited $\mathrm{H}_{2} \mathrm{~S}$ in vivo and in vitro ${ }^{32,33}$. Choi et al. reported that KRG at doses of 50-100 $\mu \mathrm{g}$ / $\mathrm{ml}$ decreased both CBS and CSE expression in human umbilical vein endothelial cells (HUVECs) $)^{34}$. In this study, the protein expression levels of CBS and CSE determined by immunoblotting (Fig. 3a) were increased by administration of high-dose KRG. That difference is considered because the KRG dose within the recommended range for pharmacological effects significantly lowered the expression of inflammatory mediators.

An investigation of the process implicated in $\mathrm{H}_{2} \mathrm{~S}$-induced inflammation and ROS, determined that high $\mathrm{H}_{2} \mathrm{~S}$ concentrations over a short period of time triggered the toxicity of $\mathrm{H}_{2} \mathrm{~S}$ via the inhibition of mitochondrial 
cytochrome c oxidase and mitochondrial respiration ${ }^{35}$. Another study reported that administration of $500 \mu \mathrm{M}$ NaHS could increase ROS formation through the inhibition of cytochrome c oxidase and the depletion of GSH in rat primary hepatocytes, which could lead to hepatotoxicity ${ }^{36} . \mathrm{H}_{2} \mathrm{~S}$ exposure activated the NF- $\mathrm{B}$ pathway, resulting in an increase in the protein levels of NF- $\kappa B$, TNF- $\alpha$, and IL-10. These results collectively indicate that $\mathrm{H}_{2} \mathrm{~S}$ can induce oxidative stress via redox homeostasis disorders in the liver. Tan et al. (2017) reported $\mathrm{H}_{2} \mathrm{~S}$ levels in wild-type mice liver tissue of approximately $1 \mu \mathrm{M} / \mathrm{L}^{37}$, which is similar to our results for the control group $(1.43 \mu \mathrm{M} / \mathrm{L})$ (Fig. 3b). Another study reported that 5-week HFD in mice induced a significant increase in hepatic $\mathrm{H}_{2} \mathrm{~S}$ production, which was associated with elevated levels of CBS and CSE expression ${ }^{31}$.

$\mathrm{H}_{2} \mathrm{~S}$ also reportedly stimulates gluconeogenesis and glycogenolysis, but inhibits glycogenesis and glycolysis, contributing to increased levels of glucose in the liver ${ }^{18}$. Overexpressed CSE in HepG2 cells stimulates $\mathrm{H}_{2} \mathrm{~S}$ generation, resulting in attenuated glycogen storage. The signaling pathways for $\mathrm{H}_{2} \mathrm{~S}$ are closely associated with gluconeogenesis and glucose production. These findings were supported by our immunoblotting results for glucose metabolism, including pAMPK and PEPCK (Fig. 3c). $\mathrm{H}_{2} \mathrm{~S}$ activates PEPCK by strengthening the glucocorticoid receptors and blocking AMPK activity ${ }^{18}$ and stimulates G6Pase and FDP expression through key gluconeogenic transcription factor $S$-sulfhydrating PGC- $1 \alpha^{19}$. Furthermore, other studies have reported that lower levels of $\mathrm{H}_{2} \mathrm{~S}$ are related to liver dysfunction and result in hepatic fibrosis and cirrhosis, whereas higher levels of $\mathrm{H}_{2} \mathrm{~S}$ strengthen insulin resistance and diabetes ${ }^{38,39}$.

In conclusion, we demonstrated that high $\mathrm{H}_{2} \mathrm{~S}$ levels may be central to liver injury and elevated glucose levels may be due to sub-chronic administration of high-dose KRG (Fig. 4). This toxic mechanism is also associated with increased protein expression of CBS and CSE in the liver. In this study, KRG-induced liver toxicity was observed for the first time, and the results help increase our understanding of the biological mechanisms underlying KRG toxicity. Nonetheless, additional research under different physiological conditions is required to further delineate the mechanisms involved in ginseng toxicity.

\section{Methods}

Study design. The male C57BL/6 N mice were obtained from Orient Co. (Seongnam, Korea) and randomly housed at 4 mice per cage. The mice were acclimated for 1 week under controlled laboratory conditions (temperature of $22 \pm 2{ }^{\circ} \mathrm{C}$, humidity of $55 \pm 5 \%$, and 12-h light/dark cycle) before the experiments, and fed standard rodent chow and tap water ad libitum. The mice (mean body weight $20.3 \pm 0.6 \mathrm{~g}$ ) were randomly divided into 4 groups ( 6 mice per group) before administration of KRG. Daily oral administration of KRG in the treatment groups $(0.5,1.0$, and $2.0 \mathrm{~g} / \mathrm{kg})$ took place over 4 weeks. The KRG extract used in this study was obtained from Punggi Ginseng Cooperative Association (Punggi, Korea) and was prepared using a traditional process that involves repeatedly steaming and drying the roots, hot-water extraction, and concentration ${ }^{27}$. The 13 ginsenosides Rb1, Rb2, Rc, Rd, Re, Rf, 20(S)-Rh1, 20(S)-Rh2, Rg1, 20(S)-Rg3, F1, F2, and compound K were absolutely quantified by LC-MS/MS ${ }^{23}$. The mice fasted for $12 \mathrm{~h}$ with free access to water before sacrifice to obtain blood and liver tissue. All animal experiments and methods were conducted in accordance with the guidelines of the Institutional Animal Care and Use Committee of Kyungpook National University and carried out in compliance with the ARRIVE guidelines.

KRG preparation. We were provided with ginseng products by Punggi Ginseng Cooperative Association (Punggi, Korea). Thirteen ginsenosides were prepared according to a traditional routine process ${ }^{40,41}$. KRG is processed by placing the cleaned and sorted ginseng on bamboo or wooden shelves in a closed steam chamber and applying steam slowly. Steaming lasted approximately 50-90 min, depending on the size of the ginseng, until it was thoroughly prepared. Then the ginseng was transferred outdoors to cool and remove the moisture. Finally, the ginseng is moved to the baking room and baked at a low temperature until proper dryness is achieved.

Serum chemistry. Blood samples were collected from the inferior vena cava and maintained at room temperature. The blood was centrifuged for $15 \mathrm{~min}$ at $4000 \times \mathrm{g}$ to obtain the serum samples. These samples were stored at $-80{ }^{\circ} \mathrm{C}$ until they were tested. Serum parameters including liver function levels and lipid parameters were analyzed at the Hoseo Toxicological Research Center (Asan, Chungcheongnam-do, Korea) using a Hitachi 7020 Chemistry Analyzer (Hitachi, Tokyo, Japan).

Liver sample preparation for proteomic analysis. Liver tissues $(n=3)$ of mice from each group were washed twice with cold phosphate-buffered saline and homogenized to extract the protein with $1 \%$ SDS buffer (1\% SDS, $2 \mathrm{mM}$ EDTA, $10 \mathrm{mM}$ Tris-HCl, pH 7.5) containing a protease inhibitor cocktail (Thermo Fisher Scientific Inc., Rockford, IL). The liver homogenates were centrifuged at $12,000 \times \mathrm{g}$ for $10 \mathrm{~min}$ at $4{ }^{\circ} \mathrm{C}$ and the supernatants were transferred to new tubes. All samples from each group were pooled to minimize sample biological variation. Protein was reduced with $15 \mathrm{mM}$ dithiothreitol and incubated at $56^{\circ} \mathrm{C}$ for $30 \mathrm{~min}$ and then alkylated with $15 \mathrm{mM}$ iodoacetamide at room temperature for $30 \mathrm{~min}$ in the dark. To purify the protein, $10 \%$ trichloroacetic acid was added to the protein samples and incubated for $4 \mathrm{~h}$ at $4{ }^{\circ} \mathrm{C}$. The protein pellets were then washed twice with ice-cold acetone. The protein samples were solubilized in $100 \mathrm{mM}$ triethylammonium bicarbonate buffer; then, Trypsin (Promega, Madison, WI) was added and the samples were incubated overnight at $37^{\circ} \mathrm{C}$. After trypsin digestion, the final concentration of $1 \%$ TFA was added to stop enzyme activation. The samples were then centrifuged at $12,000 \times \mathrm{g}$ for $10 \mathrm{~min}$, the digested peptides were collected, and the $100 \mu \mathrm{g}$ samples were obtained. The samples were kept in $-80^{\circ} \mathrm{C}$ until use.

Histopathology. Sliced liver samples $(1 \mathrm{~cm} \times 1 \mathrm{~cm})$ taken from the largest lobe were fixed overnight with $10 \%$ formalin (Sigma Aldrich, St. Louis, US) in phosphate-buffered saline solution, embedded in paraffin, and 
stored at room temperature before analysis. A histological examination was performed at Histoire (Ansan, Gyeonggi-do, Korea) and Chosun University (Gwangju, Korea) to determine morphological changes. Liver tissues were stained with $\mathrm{H} \& \mathrm{E}$ and observed under 200X magnification.

TMT labeling and high $\mathrm{pH}$ fractionation. A total of $40 \mu \mathrm{g}$ of peptide from each group was labeled with 6-plex\TMT reagent (Thermo Fisher Scientific Inc., Rockford, lL), according to the manufacturer's protocol. Briefly, the labels (Control, 126; KRG $0.5 \mathrm{~g} / \mathrm{kg}, 127 ; \mathrm{KRG} 1 \mathrm{~g} / \mathrm{kg}, 128$; KRG $2 \mathrm{~g} / \mathrm{kg}, 129$ ) were dissolved in $41 \mu \mathrm{L}$ acetonitrile $(\mathrm{ACN})$ prior to labeling, and $20 \mu \mathrm{L}$ was added to each sample for incubation. After incubating for $1 \mathrm{~h}, 8 \mu \mathrm{L}$ of $5 \%$ hydroxylamine was added to stop each reaction for $15 \mathrm{~min}$. Then, the samples were combined with the dried labeled peptides using vacuum centrifugation. High $\mathrm{pH}$ fractionation (Thermo Fisher Scientific Inc., Rockford, IL) was performed with 8 different buffers, according to the manufacturer's protocol in order to increase proteome coverage. Briefly, 8 different buffers of $0.1 \%$ trimethylamine solution, including $5,7.5$, $10,12.5,15,17.5,20$, and $50 \%$ ACN were used to elute the TMT-labeled peptides. All fractioned samples were cleaned with C18 Ziptips (Millipore, Billerica, MA), according to the manufacturer's protocol. All samples were prepared for duplicate LC-MS/MS analysis.

LC-MS/MS analysis. The digested samples were analyzed using a LTQ Velos-Orbitrap Mass Spectrometer (Thermo Fisher Scientific, Waltham, MA), linked online to nanoLC. Eight fractions from TMT-labeled peptides $(2 \mu \mathrm{g})$ were directly injected into a nanospray ionization source equipped with a hand-made reverse-phase analytical column (Proteo C12 $4 \mu \mathrm{m}$ beads, $90 \AA$ pore size, phenomenex Torrance, CA, USA). The separation with nanoLC was performed using a flow rate of $300 \mathrm{~nL} / \mathrm{min}$. For data-dependent acquisition, a 60 -min binary linear gradient was used. The gradients started with $3 \%$ solvent B (100\% ACN with $0.1 \%$ FA) for 2 min, 3-21\% solvent B for $50 \mathrm{~min}$, and 21-90\% solvent B for 3 min using Eksigent nanoLC (SCIX, Redwood city, CA). The LTQ velos orbitrap was run set at $2.0 \mathrm{kV}$ electrospray source voltage and data-dependent MS/MS mode. For the MS settings, the MS scan (300-1800 m/z) was set to have a resolution of 60,000 and $1.0 \times 10^{6}$ automated gain control (AGC). Normalized collision energy at $40 \%$ with HCD mode, $0.1 \mathrm{~ms}$ activation time, and first mass fixed at $100 \mathrm{~m} / \mathrm{z}$. This was followed by collision-induced dissociation MS/MS scans for up to the 10 most abundant ions, with a resolution of $7500,1.0 \times 10^{6} \mathrm{AGC}$ and $100 \mathrm{~ms}$ maximum ion injection time.

Identification of differentially expressed proteins. MaxQuant 1.5 integrated with the Andromeda search engine was used for searching MS/MS data ${ }^{42}$. Tandem mass spectra were analyzed against a total of 51,444 mouse sequences in the UniProtKB/Swiss-Prot database concatenated with the reverse decoy database and common contaminants. Trypsin was specified as a cleavage enzyme, allowing for two missed cleavages. Carbamidomethylation on cysteine was set as a fixed modification, whereas methionine oxidation and protein $\mathrm{N}$-term acetylation were set as variable modifications. For protein quantification through TMT labeling, we calculated the ratio of reporter ions using the TMT 6-plex method at MaxQaunt 1.5. All other parameters in MaxQuant were set to default values. We excluded contamination to obtain a high-quality protein list. The differentially expressed peptides include peptides with FDR $\leq 0.01$, score $>40$, and absolute log2-fold-changes 0.58 ( 1.5 -fold), which are calculated from the TMT reagent ion reporter ratio of different-dose KRG groups, compared to the control group. DEPs are defined as proteins with a fold change greater than 1.5 or less than 0.666 in terms of relative abundance.

Bioinformatics. The list of GO analysis, $\mathrm{KEGG}^{43}$, and InterPro were classified according to the top 5 enrichment annotations among the upregulated proteins. The basis for calculating enrichment values for the mouse proteins was achieved using DAVID 6.7 online software (https://david.ncifcrf.gov/ $)^{44,45}$. The high enrichment was categorized by the EASE score and a modified Fisher's exact test $p$ value below 0.05 . Visualizations, such as the volcano plot, for proteomics results were drawn using Perseus 1.6 (http://www.perseus-framework.org) ${ }^{46}$.

Immunoblot analysis. To confirm the CSE and CBS protein expression levels, $10 \mu \mathrm{g}$ protein samples lysed in RIPA buffer (25 mM Tris-HCl, pH 7.6, $150 \mathrm{mM} \mathrm{NaCl}, 1 \%$ NP-40, 1\% sodium deoxycholate, $0.1 \%$ SDS; Thermo Scientific) were prepared from the livers taken from each group. The proteins were resolved on a $10 \%$ gel and transferred to a polyvinylidine difluoride membrane (Millipore, Bedford, MA, USA). The membrane was then incubated overnight with primary antibodies at $4{ }^{\circ} \mathrm{C}$. Anti-CSE (MBS2015730) was obtained from My BioSource (San Diego, CA, USA), and anti-CBS (\#14782), anti-TNF- $\alpha$ (\#6945), anti-IL-6 (\#12153), anti-phospho-

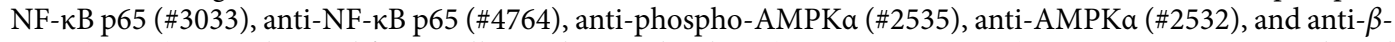
actin (\#4967) were obtained from Cell Signaling Technology (Canvers, MA, USA). Anti-PEPCK was purchased from Santa Cruz (Dallas, TX, USA). The secondary antibodies were incubated for $1 \mathrm{~h}$ at room temperature. Anti-Rabbit (\#7074) and anti-Mouse (\#7076) were obtained from Cell signaling Technology (Canvers, MA, USA). Bands were detected using an enhanced chemiluminescence system (Amersham, Buckinghamshire, UK).

$\mathrm{H}_{2}$ S assays. A total of $20 \mathrm{mg}$ of homogenated liver samples were centrifuged for $15 \mathrm{~min}$ at $1500 \times g$ with cold PBS. The supernatant was then transferred into new tubes and assayed immediately or aliquoted and stored at $-80^{\circ} \mathrm{C} . \mathrm{H}_{2} \mathrm{~S}$ (Kamiya biomedical company, Seattle, WA, USA) was measured as per the manufacturer's protocols. Briefly, the samples and standards were incubated at $37^{\circ} \mathrm{C}$ for $1 \mathrm{~h}$ after mixing with $50 \mu \mathrm{L}$ of conjugate solution in antibody pre-coated plates. Substrate solutions were then reacted in the plate for 10-15 min after 5 washes with wash buffer. The stop solution was added and absorbance was measured at $450 \mathrm{~nm}$. All samples were quantified and standardized to the same protein concentration using the BCA kit and calculated per mg of pro- 
tein after performing the $\mathrm{H}_{2} \mathrm{~S}$ assay. Standard curves were constructed by plotting the average OD to generate a four-parameter logistic curve-fit. The $\mathrm{H}_{2} \mathrm{~S}$ activity levels of all samples were calculated by adjusting the standard curve and divided by protein concentration.

Statistical analysisStatistical analysis. Statistical analyses were conducted using the SPSS Statistics 23 software package (IBM Corp., Armonk, NY). All data were expressed as mean \pm standard error. For variance homogeneity, the data were analyzed using the F-value in Levene's test. If it was not significant, the ANOVA test was conducted, followed by Bonferroni's multiple comparisons test. Differences between groups were considered significant at $p<0.05\left(^{\star}\right), p<0.01\left(^{* *}\right)$, and $p<0.001\left(^{* * *}\right)$.

Ethical statement. All experimental protocols were approved by a KNU Industry Foundation for Safety Management Systems at Kyungpook National University (Approved No. KNU-2017-0089-1).

\section{Data availability}

The mass spectrometry proteomics data have been deposited to the ProteomeXchange Consortium via the PRIDE partner repository with the dataset identifier PXD023731.

Received: 14 April 2020; Accepted: 19 May 2021

Published online: 11 June 2021

\section{References}

1. Yun, T. K. Brief introduction of Panax ginseng C.A. Meyer. J Korean Med Sci $16^{*}(2001)$.

2. Park, J. D., Rhee, D. K. \& Lee, Y. H. Biological activities and chemistry of saponins from panax ginseng C A. Meyer. Phytochem. Rev. 4, 159-175. https://doi.org/10.1007/s11101-005-2835-8 (2005).

3. Kim, M. H. et al. Antioxidant and hepatoprotective effects of fermented red ginseng against high fat diet-induced hyperlipidemia in rats. Lab. Anim. Res. 32, 217-223. https://doi.org/10.5625/lar.2016.32.4.217 (2016).

4. Liu, R. et al. Ginseng (Panax ginseng Meyer) oligopeptides protect against binge drinking-induced liver damage through inhibiting oxidative stress and inflammation in rats. Nutrients https://doi.org/10.3390/nu10111665 (2018).

5. Lu, S. et al. Ginsenoside compound K protects human umbilical vein endothelial cells against oxidized low-density lipoproteininduced injury via inhibition of nuclear factor- $\kappa \mathrm{B}$, p38, and JNK MAPK pathways. J. Ginseng Res. 43, 95-104. https://doi.org/10. 1016/j.jgr.2017.09.004 (2019).

6. Chan, P. \& Fu, P. P. Toxicity of Panax Genseng-an herbal medicine and dietary supplement. J. Food Drug Anal. 15, 416-427 (2007).

7. Cho, J. H., Kim, H. K., Baik, S. O., Choi, S. Y. \& Lee, J. Y. Single- and repeated-dose toxicities of aloe fermentation products in rats. Lab. Anim. Res. 27, 235-244. https://doi.org/10.5625/lar.2011.27.3.235 (2011).

8. Lee, J. Y. et al. Vascular smooth muscle dysfunction and remodeling induced by ginsenoside $\mathrm{Rg} 3$, a bioactive component of ginseng. Toxicol. Sci. Off. J. Soc. Toxicol. 117, 505-514. https://doi.org/10.1093/toxsci/kfq201 (2010).

9. Jung, Y. et al. Ginsenoside Rg3 disrupts actin-cytoskeletal integrity leading to contractile dysfunction and apoptotic cell death in vascular smooth muscle. Food Chem. Toxicol. Int. J. Publ. Br. Ind. Biol. Res. Assoc. 118, 645-652. https://doi.org/10.1016/j.fct.2018. 06.015 (2018).

10. Paredes-Flores, M. A. \& Mohiuddin, S. S. in StatPearls (2020).

11. Teschke, R., Eickhoff, A., Brown, A. C., Neuman, M. G. \& Schulze, J. Diagnostic biomarkers in liver injury by drugs, herbs, and alcohol: tricky dilemma after EMA correctly and officially retracted letter of support. Int. J. Mol. Sci. https://doi.org/10.3390/ijms2 1010212 (2019).

12. Park, S. H. Implication of liver enzymes in viral and alcoholic liver diseases. Korean J. Hepatol. 17, 237-242 (2011).

13. Kim, T. W. Ginseng for liver injury: friend or foe?. Medicines (Basel). https://doi.org/10.3390/medicines3040033 (2016).

14. Rinaldi, L. et al. Hydrogen sulfide prevents apoptosis of human PMN via inhibition of p38 and caspase 3. Lab. Invest. 86, 391-397 (2006).

15. Baskar, R., Li, L. \& Moore, P. K. Hydrogen sulfide-induces DNA damage and changes in apoptotic gene expression in human lung fibroblast cells. FASEB J. 21, 247-255 (2007).

16. Norris, E. J., Culberson, C. R., Narasimhan, S. \& Clemens, M. G. The liver as a central regulator of hydrogen sulfide. Shock 36, 242-250 (2011).

17. Kimura, H. Production and physiological effects of hydrogen sulfide. Antioxid. Redox Signal. 20, 783-793 (2014).

18. Zhang, L. et al. Hydrogen sulfide impairs glucose utilization and increases gluconeogenesis in hepatocytes. Endocrinology 154, 114-126. https://doi.org/10.1210/en.2012-1658 (2013).

19. dUntereiner, A. A., Wang, R., Ju, Y. \& Wu, L. Decreased gluconeogenesis in the absence of cystathionine gamma-lyase and the underlying mechanisms. Antioxid. Redox Signal. 24, 129-140. https://doi.org/10.1089/ars.2015.6369 (2016).

20. Szabó, C. Hydrogen sulphide and its therapeutic potential. Nat. Rev. Drug Discov. 6, 917-935. https://doi.org/10.1038/nrd2425 (2007).

21. Jin, Y. R. et al. Antithrombotic and antiplatelet activities of Korean red ginseng extract. Basic Clin. Pharmacol. Toxicol. 100, $170-175$. https://doi.org/10.1111/j.1742-7843.2006.00033.x (2007).

22. Nair, A. B. \& Jacob, S. A simple practice guide for dose conversion between animals and human. J. Basic Clin. Pharm. 7, 27-31. https://doi.org/10.4103/0976-0105.177703 (2016).

23. Jung Jae, J., Pil Joung, C. \& Sangkyu, L. Simultaneous quantification of 13 ginsenosides by LC-MS/MS and its application in diverse ginseng extracts. Mass Spectrom. Lett. 9, 41-45 (2018).

24. Seo, H. W., Suh, J. H., Kyung, J.-S., Jang, K. H. \& So, S.-H. Subacute oral toxicity and bacterial mutagenicity study of a mixture of Korean red Ginseng ( Panax ginseng C. A. Meyer) and Salvia plebeia . R. Br. Extracts. Toxicol. Res. 35, 215-224. https://doi.org/ 10.5487/TR.2019.35.3.215 (2019).

25. Park, S.-J. et al. Subchronic oral toxicity study of Korean red ginseng extract in Sprague-Dawley rats with a 4-week recovery period. Regul. Toxicol. Pharmacol. 92, 83-93. https://doi.org/10.1016/j.yrtph.2017.11.007 (2018).

26. Zhao, W., Zhang, J., Lu, Y. \& Wang, R. The vasorelaxant effect of $\mathrm{H}(2) \mathrm{S}$ as a novel endogenous gaseous K(ATP) channel opener. EMBO J 20, 6008-6016 (2001).

27. Hong, M. et al. Anti-inflammatory and antifatigue effect of Korean Red Ginseng in patients with nonalcoholic fatty liver disease. J. Ginseng Res. 40, 203-210. https://doi.org/10.1016/j.jgr.2015.07.006 (2016).

28. Nam, S. J. et al. Effect of red ginseng extract on the pharmacokinetics and efficacy of metformin in streptozotocin-induced diabetic rats. Pharmaceutics 10, 80 (2018). 
29. Gao, Y. L. et al. Subchronic toxicity studies with ginsenoside compound $\mathrm{K}$ delivered to dogs via intravenous administration. Food Chem. Toxicol. 49, 1857-1862. https://doi.org/10.1016/j.fct.2011.05.003 (2011).

30. Lin, K. et al. Ginseng-related drug-induced liver injury. Case Rep. Gastroenterol. 12, 439-446. https://doi.org/10.1159/000490525 (2018).

31. Sawa, T., Kumagai, Y. \& Akaike, T. Regulation of Redox Signaling by a Nitrated Nucleotide and Reactive Cysteine Persulfides. 231-235 (2017). https://doi.org/10.1016/b978-0-12-804273-1.00017-x

32. Lee, J. S., Kwon, K. A., Jung, H. S., Kim, J. H. \& Hahm, K. B. Korea red ginseng on Helicobacter pylori-induced halitosis: newer therapeutic strategy and a plausible mechanism. Digestion 80, 192-199 (2009).

33. Lee, S. et al. Korean red ginseng ameliorated experimental pancreatitis through the inhibition of hydrogen sulfide in mice. Pancreatology 16, 326-336 (2016).

34. Choi, K. S. et al. Inhibition of hydrogen sulfide-induced angiogenesis and inflammation in vascular endothelial cells: potential mechanisms of gastric cancer prevention by Korean Red Ginseng. J. Ginseng Res. 36, 135-145 (2012).

35. Reiffenstein, R. J., Hulbert, W. C. \& Roth, S. H. Toxicology of hydrogen sulfide. Annu. Rev. Pharmacol. Toxicol. 32, 109-134 (1992).

36. Truong, D. H., Eghbal, M. A., Hindmarsh, W., Roth, S. H. \& O’Brien, P. J. Molecular mechanisms of hydrogen sulfide toxicity. Drug Metab. Rev. 38, 733-744. https://doi.org/10.1080/03602530600959607 (2006).

37. Tan, B. et al. New method for quantification of gasotransmitter hydrogen sulfide in biological matrices by LC-MS/MS. Sci. Rep. 7, 46278. https://doi.org/10.1038/srep46278 (2017).

38. Wang, R. Physiological implications of hydrogen sulfide: a whiff exploration that blossomed. Physiol. Rev. 92, 791-896 (2012).

39. Mani, S., Li, H., Yang, G., Wu, L. \& Wang, R. Deficiency of cystathionine gamma-lyase and hepatic cholesterol accumulation during mouse fatty liver development. Sci. Bull. 60, 336-347. https://doi.org/10.1007/s11434-014-0722-7 (2015).

40. Lee, S. M. et al. Characterization of Korean Red Ginseng (Panax ginseng Meyer): history, preparation method, and chemical composition. J. Ginseng Res. 39, 384-391. https://doi.org/10.1016/j.jgr.2015.04.009 (2015).

41. Institute., B. R. Exploration of Ginseng Plantation and Processing. SUN TEN QUARTERLY NEWSLETTER 2, http://www.sunte nglobal.com/news/img/QNSpring09.pdf (2018).

42. Cox, J. \& Mann, M. MaxQuant enables high peptide identification rates, individualized p.p.b.-range mass accuracies and proteomewide protein quantification. Nat. Biotechnol. 26, 1367-1372. https://doi.org/10.1038/nbt.1511 (2008).

43. Kanehisa, M., Furumichi, M., Sato, Y., Ishiguro-Watanabe, M. \& Tanabe, M. KEGG: integrating viruses and cellular organisms. Nucleic Acids Res. 49, D545-d551. https://doi.org/10.1093/nar/gkaa970 (2021).

44. da Huang, W., Sherman, B. T. \& Lempicki, R. A. Systematic and integrative analysis of large gene lists using DAVID bioinformatics resources. Nat. Protoc. 4, 44-57. https://doi.org/10.1038/nprot.2008.211 (2009).

45. da Huang, W., Sherman, B. T. \& Lempicki, R. A. Bioinformatics enrichment tools: paths toward the comprehensive functional analysis of large gene lists. Nucleic Acids Res. 37, 1-13. https://doi.org/10.1093/nar/gkn923 (2009).

46. Tyanova, S. et al. The Perseus computational platform for comprehensive analysis of (prote)omics data. Nat. Methods 13, 731-740. https://doi.org/10.1038/nmeth.3901 (2016).

\title{
Acknowledgements
}

This work was supported by the Korea Institute of Planning and Evaluation for Technology in Food, Agriculture, Forestry and Fisheries (IPET) through the Export Promotion Technology Development Program, funded by the Ministry of Agriculture, Food, and Rural Affairs (Grant Number 316017-3), by the National Research Foundation of Korea (NRF) grant (NRF-2018R1D1A1A02043591) by the Korean government and by the Basic Science Research Capacity Enhancement Project through the Korea Basic Science Institute (National research Facilities and Equipment Center) grant funded by the Ministry of Education (Grant No. 2019R1A6C1010001).

\section{Author contributions}

A.Y.N. and S.K.L. wrote the main manuscript and J.J.J. and P.C. conducted the animal experiments. O.K.K. Y.G. contributed to the proteomics analysis. K.M.K. and S.H.K. prepared Fig. 3. J.H.K. contributed with data interpretation. All authors reviewed the manuscript.

\section{Competing interests}

The authors declare no competing interests.

\section{Additional information}

Supplementary Information The online version contains supplementary material available at https:/doi.org/ 10.1038/s41598-021-91664-8.

Correspondence and requests for materials should be addressed to S.H.K. or S.L.

Reprints and permissions information is available at www.nature.com/reprints.

Publisher's note Springer Nature remains neutral with regard to jurisdictional claims in published maps and institutional affiliations.

\begin{abstract}
Open Access This article is licensed under a Creative Commons Attribution 4.0 International format, as long as you give appropriate credit to the original author(s) and the source, provide a link to the Creative Commons licence, and indicate if changes were made. The images or other third party material in this article are included in the article's Creative Commons licence, unless indicated otherwise in a credit line to the material. If material is not included in the article's Creative Commons licence and your intended use is not permitted by statutory regulation or exceeds the permitted use, you will need to obtain permission directly from the copyright holder. To view a copy of this licence, visit http://creativecommons.org/licenses/by/4.0/.
\end{abstract}

(C) The Author(s) 2021 